Final Report, DOE Interagency Agreement No. DE-AI02-05ER63958

\title{
From Measurements to Models: Cross-Comparison of Measured and Simulated Behavioral States of the Atmosphere
}

Anthony D. Del Genio (NASA Goddard Institute for Space Studies), PI

Forrest M. Hoffman and William W. Hargrove, Jr. (DOE/ORNL), Co-Is

$10 / 22 / 2007$

1.) Introduction

The DOE Atmospheric Radiation Measurement Program (ARM) sites and the ARM Mobile Facility (AMF) were constructed to make measurements of the atmosphere and radiation system in order to quantify deficiencies in the simulation of clouds within models and to make improvements in those models. While the measurement infrastructure of ARM is well-developed and a model parameterization testbed capability has been established, additional effort is needed to develop statistical techniques which permit the comparison of simulation output from atmospheric models with actual measurements. Our project established a new methodology for objectively comparing ARM measurements to the outputs of leading global climate models and reanalysis data. The quantitative basis for this comparison is provided by a statistical procedure which establishes an exhaustive set of mutually-exclusive, recurring states of the atmosphere from sets of multivariate atmospheric and cloud conditions, and then classifies multivariate measurements or simulation outputs into those states. Whether measurements and models classify the atmosphere into the same states at specific locations through time provides an unequivocal comparison result. Times and locations in both geographic and state space of modelmeasurement agreement and disagreement will suggest directions for the collection of additional measurements at existing sites, provide insight into the global representativeness of the current ARM sites (suggesting locations and times for use of the AMF), and provide a basis for improvement of models. Below we describe the results of two projects completed under the auspices of this Interagency Agreement that use the cluster analysis approach.

2.) Simulations of anthropogenic climate changes in the hydrological cycle

Changes in Earth's climate in response to atmospheric greenhouse gas buildup impact the health of terrestrial ecosystems and the hydrologic cycle. The environmental conditions influential to plant and animal life are often mapped as ecoregions, which are land areas having similar combinations of environmental characteristics. This idea is extended to establish regions of similarity with respect to climatic characteristics that evolve through time using a quantitative statistical clustering technique called Multivariate Spatio-Temporal Clustering (MSTC). MSTC was applied to the monthly time series output from a fully coupled general circulation model (GCM) called the Parallel Climate Model (PCM). Results from an ensemble of five 99-yr Business-As-Usual (BAU) transient simulations from 2000 to 2098 were analyzed (Hoffman et al., 2005). 
MSTC establishes an exhaustive set of recurring climate regimes that form a "skeleton" through the "observations" (model output) throughout the occupied portion of the climate phase space formed by the characteristics being considered. MSTC facilitates direct comparison of ensemble members and ensemble and temporal averages since the derived climate regimes provide a basis for comparison. Moreover, by mapping all land cells to discrete climate states, the dynamic behavior of any part of the system can be studied by its time-varying sequence of climate state occupancy. MSTC is a powerful tool for model developers and environmental decision makers who wish to understand long, complex time series predictions of models. Strong predicted interannual trends were revealed in this analysis, including an increase in global desertification; a decrease in the cold, dry high-latitude conditions typical of North American and Asian winters; and significant warming in Antarctica and western Greenland.

Publication resulting from this work:

Hoffman, F.M., W.W. Hargrove Jr., D.J. Erickson III, and R.J. Oglesby (2005): Using clustered climate regimes to analyze and compare predictions from fully coupled general circulation models. Earth Interactions, 9, 1-27.

\section{3.) Current climate cloud regimes in ARM data and GCM simulations}

We applied the k-means clustering technique to 5 years of ARM ARSCL data at the ARM Manus and Nauru Tropical West Pacific sites, to the same 5 years of International Satellite Cloud Climatology Project (ISCCP) data for the entire tropics, and to 5 years of simulations using the GISS Model E GCM (Chen and Del Genio, 2007). The ISCCP cluster analysis produces 6 independent cloud regimes corresponding approximately to known tropical "weather states" such as deep convection, anvil clouds, midlevel cumulus congestus, isolated cirrus, trade cumulus and marine stratocumulus. These states also exist at Manus and Nauru, making them representative of the overall tropical behavior, with the exception that the stratocumulus regime, which is typical of the eastern oceans, is very infrequent in both places.

We used ARSCL to diagnose the distribution of highest cloud top heights at both sites for each cluster. This diagnostic varies relatively little from one cloud regime to another, because high thin cirrus are ubiquitous in the tropics. It is only when the full ARSCL distribution of clouds is analyzed that significant differences among the ISCCP cloud regimes become evident. Comparison to the ISCCP cloud top height distribution indicates that ISCCP systematically underestimates the highest cloud top and overestimates midlevel top clouds because it sometimes sees through high thin cirrus, cannot distinguish multilayer clouds, and sometimes uses erroneous input temperature and humidity profiles. We tested the ability of the ISCCP cloud regimes and the ARSCL vertical cloud profiles to capture systematic variations associated with the Madden-Julian Oscillation. Using $8 \mathrm{MJO}$ events in ISCCP and 4 events that also affected Manus and are visible in ARSCL data, we plotted the composite cloud regimes and structure as a function of phase relative to the MJO peak. We find that there is a systematic transition in cloud heights and cover from one phase to another that supports the idea of the "discharge-recharge" mechanism of convection height regulation by tropospheric moisture as an important process for MJO evolution. 
We also examined cloud regimes produced by clustering analysis of a new version of the GISS Model E GCM at $2^{\circ} \times 2.5^{\circ} \times 32 \mathrm{~L}$ resolution that includes diagnosed cumulus updraft velocites and upward transport of convective condensate by the updrafts. We find that the GCM produces 4 clusters (two disturbed convective states and two suppressed states); it combines 2 of the observed clusters (anvils, congestus) into a single state and does not simulate an isolated cirrus state at all. Furthermore, diagnosing the shallow convection occurrence in the GCM, we find that it is not uniquely associated with the trade cumulus cluster but occurs to some extent in all clusters. Thus, the clustering approach identifies characteristic cloud types but does not isolate specific processes in the model. Finally, we find that the GCM underpredicts midlevel clouds relative to ARSCL at Manus and Nauru. These results suggest specific targets for parameterization improvement, e.g., increased entrainment rates (which are now being tested against ARM TWP-ICE field experiment data) and a modification of how the GCM treats ice supersaturation.

Publication resulting from this work:

Chen, Y., and A.D. Del Genio (2007): Evaluation of tropical cloud regimes in observations and a general circulation model. Climate Dynamics, submitted. 